\title{
Renormalization the Energy of Elementary Excitations in Solids by the Strong (Nuclear) Interaction
}

\author{
V. G. Plekhano \\ Fonoriton Sci. Lab., Garon Ltd., Lasnamae 22 - 3, Tallinn 11413, Estonia
}

Copyright (c) 2017 by authors, all rights reserved. Authors agree that this article remains permanently open access under the terms of the Creative Commons Attribution License 4.0 International License

\begin{abstract}
The experimental evidence of renormalization the energy of the elementary excitations in solids which are differ by term of one neutron from each other by the strong (nuclear) interaction has been presented for the first time. This evidence is based on two independent results: 1) The increase exciton energy on $103 \mathrm{meV}$ is caused by the adding of one neutron (using LiD crystals instead $\mathrm{LiH}$ ones); 2) After increasing the amounts by one neutron the energy of LO phonons has decreased by $36 \mathrm{meV}$. The last one is directly seen from luminescence and scattering spectra. As far as the gravitation, electromagnetic and weak interactions are the same in both of kind crystals, it only changes the strong interaction. Therefore a logical conclusion is made that the renormalization of the energy of electromagnetic excitations (excitons, phonons) is carried out by the strong (nuclear) interaction.
\end{abstract}

Keywords Strong Interaction, Quarks, Excitons, Phonons, Quantum Chromodynamics, Quantum Electrodynamics

PACS: 12.38.-t; 12.39.-x; 14.20.-c; 71.35.-y; 78.35. c; 78.55.Hx

\section{Introduction}

Our present knowledge of physical phenomena suggests that there four types of forces between physical bodies (see, e.g. [1]):

1) gravitational;

2) electromagnetic;

3) strong;

4) weak.

Both the gravitational and the electromagnetic forces vary in strength as the inverse square of the distance and so able to influence the state of an object even at very large distances whereas the strong and the weak forces fall off exponentially and so act only at extremely short distances. The strong forces does not act on leptons (electrons, positrons, muons and neutrinos), but only on protons and neutrons (more generally, on baryons and mesons - this is the reason for the collective name hadrons). It holds protons and neutrons together to form nuclei, and is insignificant at distances greater than $10^{-15} \mathrm{~m}$ [2]. Its macroscopic manifestations are restricted up to now to radioactivity and the release of nuclear energy. The three forces which are relevant to elementary particles can be recognized in the three kinds of radioactivity: $\alpha$ - radiation is caused by the strong force, $\beta$ - radiation by the weak force, and $\gamma$ - radiation by the electromagnetic force. The characteristics of these forces are summarized in Table 1. This table given for the strength and range of the forces come from a comparison of the effects they produce on two protons. In some respect these resemble an ordinary Newtonian force between the protons, varying with the distance between them as if the force was derived from a potential function:

$$
\mathrm{V}(\mathrm{r})=\frac{\mathrm{ke}^{-r / R}}{\mathrm{r}^{n}}
$$

for some n. This is an inverse - power force which is diminished by an exponential factor at distances larger than a certain distance $\mathrm{R}$, the range of the force. The strength of the force is measured by the constant $\mathrm{k}$. The unit of strength is $\mathrm{hc} / 2 \pi$ where $\mathrm{h}$ is Planck's constant and $\mathrm{c}$ the speed of light. We should add that the weak force does not appear to be particularly weak on this reckoning: the reason for its very short range (see Table 1) rather than its intrinsic strength. Since the protons and neutrons which make up the nucleus are themselves considered to be made up of quarks are considered to be held together by the color force [2], the strong force between nucleons may be considered to be a residual color force (see, also [3, 4]). In the Standard Model, therefore the base exchange is the gluon which mediates the forces between quarks. The modern quantummechanical view of the three fundamental forces (all except gravity) is that particles of matter (fermions = neutrons, protons, electrons) do not directly interact with each other, but rather carry a charge, and exchange virtual particles (gauge bosons = photons, gluons, gravitons) which are the interaction carriers or force mediators. As can be see from Table 1, photons are the mediators of the interaction of electric charges (protons, electrons, 
positrons); and gluons are the mediators of the interaction of color charges (quarks). In our days, the accepted view is that all matter is made of quarks and leptons (see Table 2). As can be see, of the three pairs of quarks and leptons, one pair of each - the quark $\mathrm{u}$ and $\mathrm{d}$ and the leptons $\mathrm{e}^{-}$and $\nu_{e}^{-}$(electrons neutrino) - are necessary to make up the every day world, and a world which contained only these would seem to be quite possible.

The facts, summarized in the modern nuclear physics (see, e.g. $[5,1])$ allow to draw several conclusions in regard to nuclear forces, most notably that the binding energy of a nucleus is proportional to the number of nucleons and that the density of nuclear matter is approximately constant. This lead to conclude that nuclear forces have a "saturation property". It seems from the last conclusion it is enough to change the number of neutrons in nucleus to change strength of nuclear force. But the last one constitutes the main ideas of the isotope effect [3].

Below we will briefly describe the results of the optical spectroscopy of isotope - mixed solids, which differ by a term of one neutron.

\section{Experimental}

The main experimental results were obtained on a device used already in the investigations [6 - 8]. The experimental equipment consisted mainly of an home - made immersion helium cryostat and two double prism (grating) monochromators (with reciprocal dispersion $10 \AA / \mathrm{mm}$ for the wavelength $\lambda=220 \mathrm{~nm}$ ) arranged at right angle. Spectra were excited by various lines of a $400 \mathrm{~W}$ deuterium lamp and a $120 \mathrm{~W}$ mercury arc lamp (see Fig. 1).

The excitation light was felt on the entrance slit of the first monochromator and after leaving it passed through quartz windows of the immersion helium cryostat and irradiated of the crystals. The luminescence and scattering light from the samples was dispersed in another double prism (grating) monochromator and photoelectrically detected. A photoelectric registration of the optical signal was realized by a cooled photopmultiplier for ultraviolet (UV) range of the spectrum and the subsequent high - sensitivity photon - counting system with a storage facilities (computer). the signal - to - noise

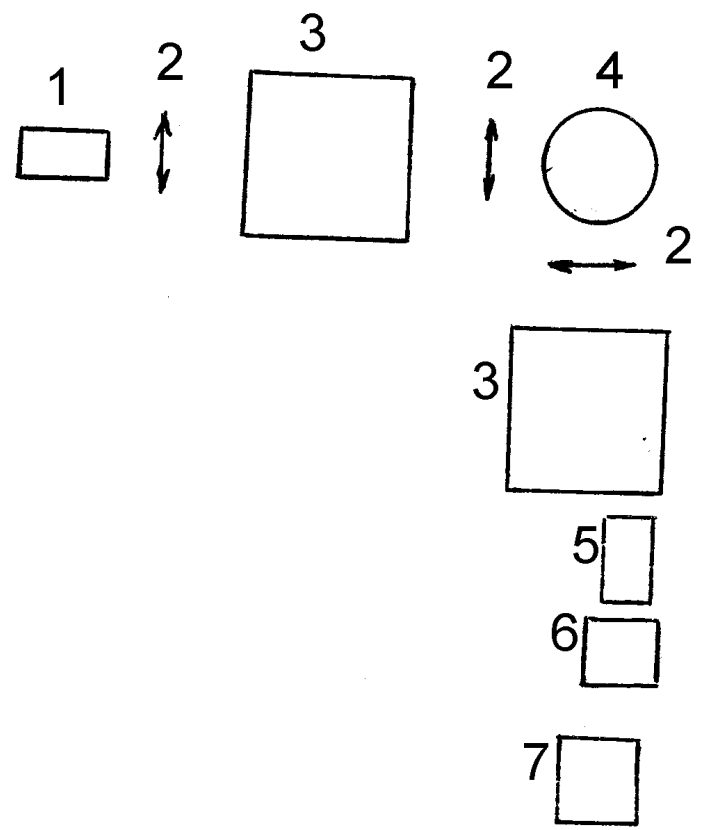

Figure 1. Scheme of experimental setup: 1 - lamp, 2 - lens, 3 - monochromator, 4 - He cryostat, 5 - photomultiplier, 6 - photon counter, 7 - personal computer.

ratio was approximately $n \cdot 10^{2}$ where $n=2 \div 5$ for different batches of crystals. The light scattering experiments were performed in the usual backscattering geomentry, at temperatures of 2 to $300 \mathrm{~K}$. To allow for the $\approx \omega^{4}$ law, a freshly cleaved of LiF crystal was used, whose dispersion of the refractive index in the spectral region 4 to $6 \mathrm{eV}$ is small. The experiments were carried out in the same way for two $(\mathrm{LiH}$ and LiD) compounds, which differ by a term of one neutron.

The single crystals of $\mathrm{LiH}$ and $\mathrm{LiD}$ were grown from the melt by the modified method of Bridgeman - Stockbarger [9]. The crystals were synthesized from ${ }^{7} \mathrm{Li}$ metal and hydrogen of $99.7 \%$ purity and deuterium of $99.5 \%$ purity. The device or LIH (LiD) single crystals growth is shown in Fig. 2.

Table 1. The four fundamental forces.

\begin{tabular}{|c|c|c|c|c|c|c|c|}
\hline Interaction & FQ & Mass & Range (m) & RS & Spin & $\mathrm{TC}-\mathrm{S}\left(\mathrm{m}^{2}\right)$ & TTS (s) \\
\hline Strong & Gluon & 0 & $10^{-15}$ & 1 & 1 & $10^{-30}$ & $10^{-23}$ \\
\hline Weak & $\mathrm{W}^{ \pm}, \mathrm{Z}$ & $81,93 \mathrm{GeV} / \mathrm{c}^{2}$ & $10^{-18}$ & $10^{-5}$ & 1,1 & $10^{-44}$ & $10^{-8}$ \\
\hline Electromagnetic & Photon & 0 & $\infty$ & $\alpha=1 / 137$ & 1 & $10^{-33}$ & $10^{-20}$ \\
\hline Gravity & Graviton & 0 & $\infty$ & $10^{-38}$ & 2 & - & - \\
\hline
\end{tabular}

Table 2. Quarks and leptons.

\begin{tabular}{|c|c|c|}
\hline \multirow{4}{*}{ Leptons } & Family & \multirow{2}{*}{ Electric charge (e) } \\
\hline & 123 & \\
\hline & $\mathrm{e}^{-} \quad \mu^{-} \tau^{-}$ & -1 \\
\hline & $\nu_{\mathrm{e}} \quad \nu_{\mu} \quad \nu_{\mathrm{t}}$ & 0 \\
\hline Quarks & $\mathrm{u} \quad \mathrm{c} \quad \mathrm{t}$ & $2 / 3$ \\
\hline & d $\quad s \quad b$ & $-1 / 3$ \\
\hline
\end{tabular}




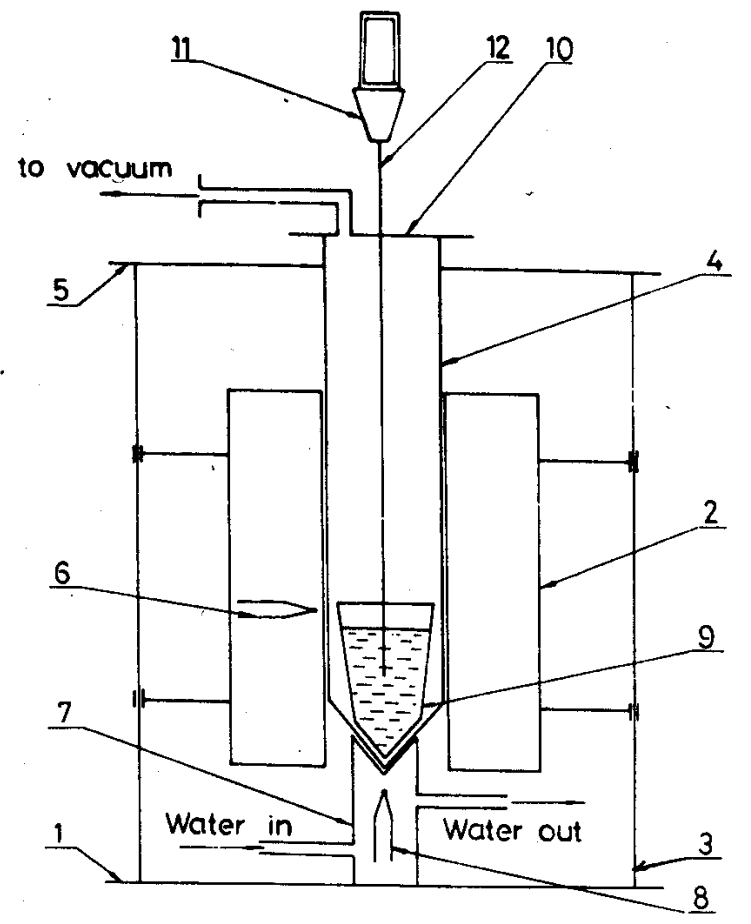

Figure 2. Scheme for growing $\mathrm{LiH}(\mathrm{LiD})$ single crystals by the modified Bridgeman - Stockbarger method (see text).

It consists of the rame (1) to which the furnace (2) is attached and is moved on the rods (3) at a rate $2-5 \mathrm{~mm} / \mathrm{h}$. The accuracy of novement is $0.02 \mathrm{~mm} / \mathrm{h}$. The filled crucible (9) is placed inside the reactor (4) which is tightly attached to the table (5). The movement of the crystalization front takes place due to the movement of the furnace, whereas the reactor is at rest. The chromel - alumel thermocouple (6) is installed into the furnace. To improve the crystalization front, a regulated temperature gradient is formed at the reactor top. The directional heat removal is made by the water cooled holder (7). The thermocouple (8) is inserted into the water - cooled holder to control the temperature of the reactor with simultaneous recording. The reactor is shut with a flange (10) provided with branch pipes to be connected with the vacuum system and a hydrogen (deuterium) inlet system. Virgin crystals fad a slightly blue - grew color, which can be attributed to nonstoichiometric excess of lithium present during the grown cycle. On annealing for several days (up to 20) at $500^{\circ} \mathrm{C}$ under $\sim 3 \mathrm{~atm}$ of hydrogen or deuterium, this color could be almost completely eliminated (Fig. 3, the more detail see [3, 9]).

Because of the high reactivity and high hygroscopy of investigated crystals an efficient protection against the atmosphere was necessing. Taking into account this circumstance, we have developed special equipment which is allowed to prepare samples with a clean surface cleaving their in the bath of helium cryostat with normal or superfluid liquid helium [6]. The samples with such surface allow to preform measurements during 15 hours. An improved of $\mathrm{LiH}(\mathrm{LiD})$ crystals were used in the present study. In spite of the identical structure of all free - exciton luminescence spectra, it is

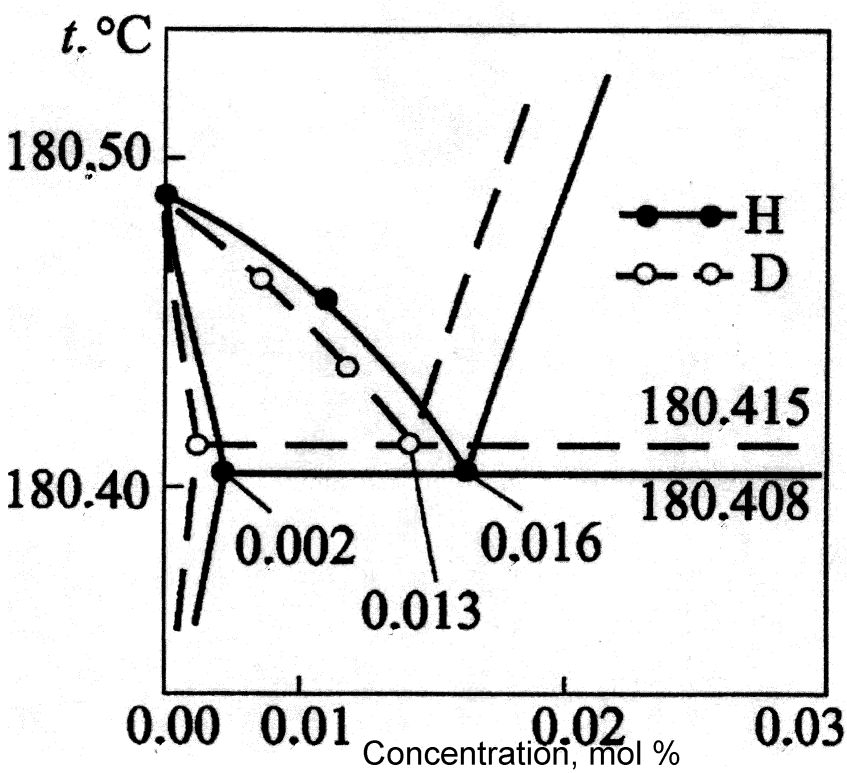

Figure 3. Diagram of the lithium metal hardened in hydrogen (deuterium) atmosphere

necessary to note a rather big variation of the luminescence intensity of the crystals from the different batches observed in experiment.

\section{Results}

It seems to be well founded [10] that most lower energy electron excitation in $\mathrm{LiH}(\mathrm{LiD})$ crystals are the large - radius excitons [11]. Under the continuous optical excitation a stationary population in the exciton states can be created. Due to the free motion in the crystal and the interaction with the crystal lattice the gas of free excitons is spread over some region of kinetic energy. However, usually only the lowest $n$ $=1 \mathrm{~S}$ (ground state) exciton is populated at low temperatures $[11,10]$. The photoluminescence emission of free excitons can take place either in resonance with the exciton absorption line (a so - called zero - phonon luminescence) or can be shifted in energy due to the simultaneous creation of phonon (phonon - assisted luminescence). In the resonant excitons emission only excitons with small wavevectors of the order of the photon wavevector $\overrightarrow{\mathrm{K}} \simeq 0$ can participate. Phonon assisted luminescence of free excitons in polar compounds is mainly due to the creation of longitudinal optical (LO) phonons [11]. Since the energy of LO phonons has a weak dependence on wavevector $\vec{q}$ [13], the spectrum of emitted photons is simply related to the initial distribution of exciton energy. Fig. 4 shows the free excitons photoluminescence in $\mathrm{LiH}$ and LiD crystals at $2 \mathrm{~K}$.

The exciton photoluminescence spectrum of $\mathrm{LiH}$ crystals cleaved in superfluid liquid helium consists of the narrow zero - phonon line and its wider phonon replicas corresponding to the radiative annihilation of excitons accompanied by excitation from one to five LO phonons. The zero - phonon emission line is almost in resonance with the reflection line 


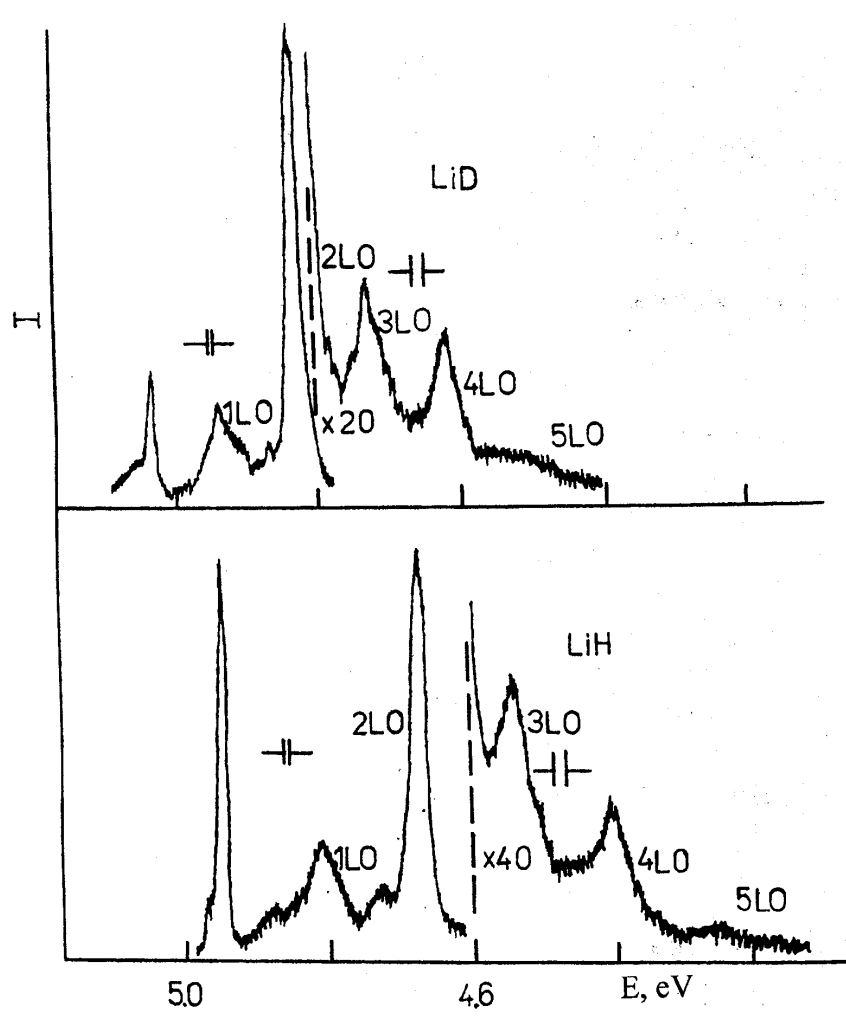

Figure 4. Photoluminescence spectra of free excitons at $2 \mathrm{~K}$ in $\mathrm{liH}$ and $\mathrm{LiD}$ crystals cleaved in superfluid helium.

in the exciton ground state $\left(\mathrm{E}_{\mathrm{n}=1 \mathrm{~S}}=4.950 \mathrm{eV}\right.$ for LiH crystals at $2 \mathrm{~K}$ [10]), which is due of the direct electron transition $\mathrm{X}_{1}-\mathrm{X}_{4}$ of the first Brillouin zone [12]. Phonons replicas form an equidistant series to the red from resonance exciton emission line. The difference in energies of these replicas, as early $[6,7,10]$ is $140 \mathrm{meV}$ which is close to the energy of LO phonons in the center of the Brillouin zone obtained in [13]. The photoluminescence spectrum of a LiD crystals with a pure surface shown also in Fig. 4 is similar in many respects to the free excitons luminescence spectrum of $\mathrm{LiH}$ crystals. However,there are some differences. These differences are related with the next manifestations:

1. The short - wavelength shift as whole of the free excitons photoluminescence spectrum of LiD crystals on 103 meV relatively the spectrum of $\mathrm{LiH}$ crystals.

2 . In the case of LiD crystals the energy difference between lines in the spectrum is on average $104 \mathrm{meV}$ corresponding to the energy of the LO phonons in the $\Gamma$ - point of the Brillouin zone [13].

At the excitation below the intrinsic absorption edge $\left(\mathrm{E}_{\mathrm{n}=1 \mathrm{~s}}=5.043 \mathrm{eV}\right.$ for LiD [10]) we have succeeded in observing the multiphonon resonance Raman scattering (RRS) with the creation of up four phonons (Fig. 5).

Indeed, the energy difference between peaks in the RRS spectrum is equal the energy of the LO phonons in the center of the Brillouin zone [13]. To pay attention the large half - width of observable lines in the RRS spectrum. As was shown in the paper [15] their half - width are always larger than of the excitation line. The proximity of the exciting light frequency to the energy of exciton transitions leads to an essential modification of the selection rules for light scattering. The presence of the second - order TO $(\Gamma)\left(\hbar \omega_{T O}(\Gamma)\right.$ $=76 \mathrm{meV}$ for $\mathrm{LiH}$ ) in the RRS spectrum may be explained by a relatively strong scattering deformation mechanism in these crystals, where, however the main mechanism, as was seen from both figures, is Frôchlich mechanism of intraband scattering. The longwavelength displacement of the excitation line frequency relatively exciton resonance a monotonic decrease the intensity of RRS spectrum as whole more than 60 - fold in both $\mathrm{LiH}$ and LiD crystals. Comparison the experimental results on the luminescence and light scattering in the crystals which differ by a term of one neutron only is allowed to the next conclusions;

1. At the adding one neutron (using LiD crystals instead $\mathrm{LiH}$ ones) is involved the increase exciton energy on 103 meV.

2. At the addition one neutron the energy of LO phonons is decreased on the $36 \mathrm{meV}$, that is direct seen from luminescence and scattering spectra.

Both observed characteristics are macroscopic.

\section{Discussion}

$\mathrm{LiH}$ (LiD) crystals with a lattice of $\mathrm{NaCl}$ type, whose parameters are close to cubic crystals, are dielectrics with band gap of $\mathrm{E}_{g}=4.992 \mathrm{eV}\left(\mathrm{E}_{g}=5.090 \mathrm{eV}\right.$ for LiD) at $2 \mathrm{~K}$ [7]. These crystals have an identical electronic structure. The energy band structure of these substances is also identical. All three kinds of forces - gravitational, electromagnetic and weak are also the same for compounds above. The difference between these substances consists out at one neutron in the nucleus of deuteron. Below we should briefly consider some peculiarities of the physics of deuteron. Nucleons can combine to make four different few - nucleon systems, the deuteron $(p+n)$, the triton $(p+2 n)$, the helion $(2 p+n)$ and the $\alpha$ - particle $(2 p+2 n)$. These particles are grouped together because they are all stable (apart from triton which has a half - life of about twelve years and so may be treated as a stable entity for most practical purposes), have no bound excited states (except the $\alpha$-particle which has two excited states at about 20 and $22 \mathrm{MeV}$ [5]), and are frequently used as projectively in nuclear reactions. Few - nucleon systems provide the simplest systems to study nuclear structure (see, e.g. [16]). The deuteron provides important information about the nucleon - nucleon interaction. As was noted, the deuteron consists of a proton and a neutron and is the only bound state of two nucleons. Its binding energy is $2.2245 \mathrm{MeV}$ and its total angular momentum $\mathbf{J}$ and parity are $1^{+}[2]$. Since the intrinsic parities of the neutron and the proton are positive parity of the deuteron implies that the relative orbital angular momentum of the neutron and the proton must be even. If the orbital angular momentum $\mathrm{L}$ is a good quantum number, states with lower orbital angular momentum generally have lower energy than states with higher angular momentum, and so we expect the ground state of the deuteron to have orbital angular momentum $\mathrm{L}=0$, so that it is in an $\mathrm{S}$ state. Then, if the spins of the proton and the neutron in the deuteron are 


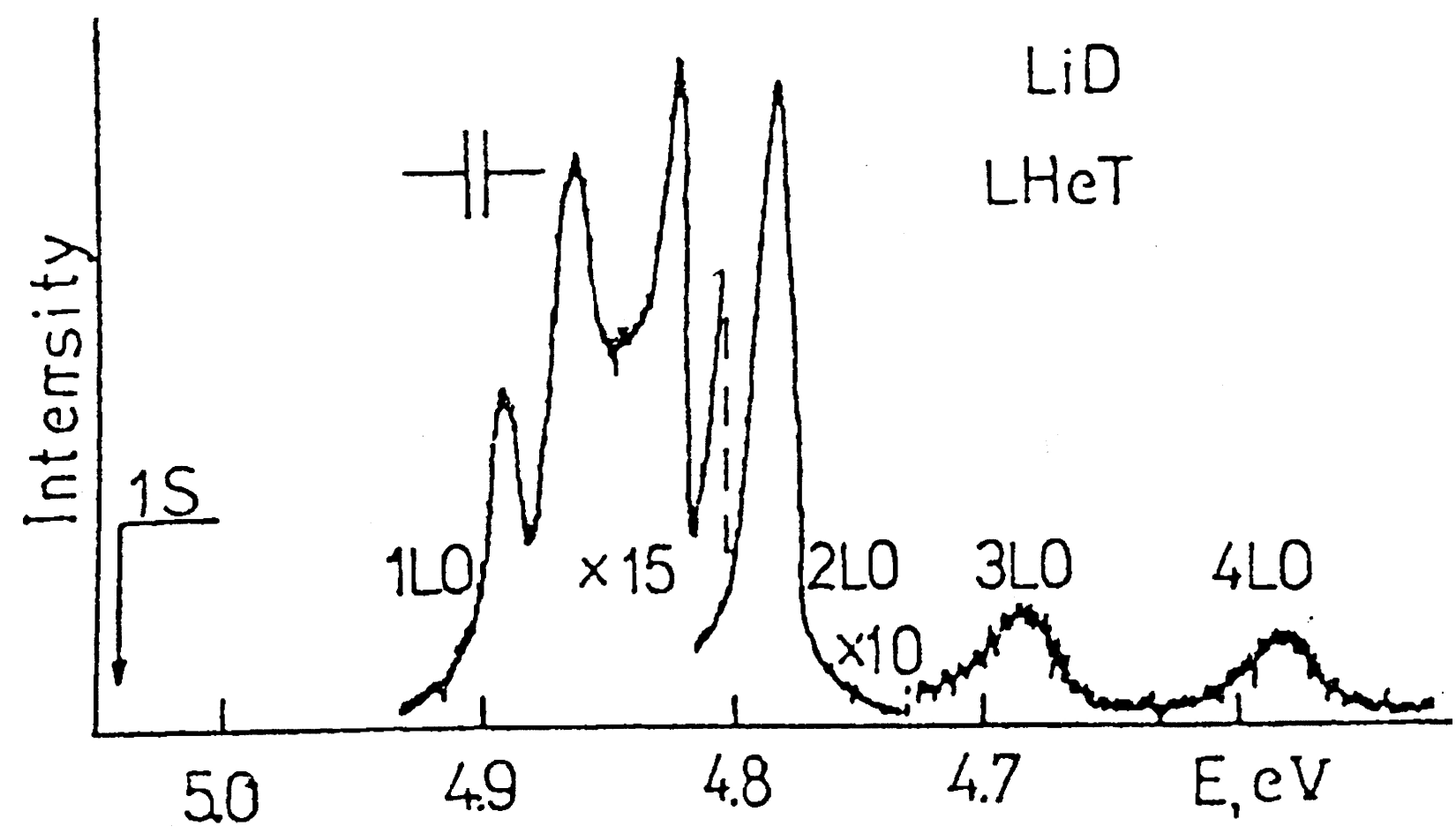

Figure 5. Resonant Raman scattering spectr of a LiD crystals at the excitation $\mathrm{E}=4.992 \mathrm{eV}$ at $4.2 \mathrm{~K}$.

parallel, we expect the magnetic moment of the deuteron to be approximately the sum of the magnetic moments of the proton and neutron, namely $\mu_{\mathrm{p}}+\mu_{\mathrm{n}}=(2.793-1.913) \mu_{\mathrm{N}}=$ $0.880 \mu_{\mathrm{N}}\left(\mu_{\mathrm{N}}=\frac{\mathrm{e} \hbar}{2 \mathrm{~m}_{\mathrm{p}}}\right)$ [5]. If, however, the spins are anti - parallel, we expect it to be $(2.793+1.913) \mu_{\mathrm{N}}=4.706 \mu_{\mathrm{N}}$. Experimentally it is $0.857 \mu_{\mathrm{N}}[5,16]$ so the spins of the proton and neutron are parallel and so the total spin $\mathrm{S}$ of the deuteron is one, since $\mathrm{J}=\mathrm{L}+\mathrm{S}, \mathrm{J}=1$. The small but definite difference between $\mu_{\mathrm{d}}=0.857 \mu_{\mathrm{N}}$ and $\mu_{\mathrm{p}}+\mu_{\mathrm{n}}=0.880 \mu_{\mathrm{N}}$ is due, as will shown below, to tensor character of strong forces in deuteron. We thus conclude that the ground state of deuteron is a triplet $\mathrm{S}$ state. However this cannot the whole story because $\mathrm{S}$ states are spherically symmetrical and thus have no quadrupole moment. This is contradict to experiments. Experimentally the deuteron has a positive quadrupole moment of $0.29 \mathrm{fm}^{2}$ [16]. The deviation of the actual deuterium moment from the $\mathrm{S}$ state moment can be explained if it assumed that the deuteron ground state is a superposition of $\mathrm{S}$ and $\mathrm{D}$ states. Part of the time, the deuteron has orbital angular momentum $\mathrm{L}=2$. Independent evidence for this fact comes from the observation that, as was shown above, the deuteron has a small, but finite, quadrupole moment (see, also [17]). As is well - known, the electric quadrupole moment measures the deviation of a charge distribution from sphericity [3]. For a spherical nucleus the expectations values of the squares of distance from the center to the surface along $\mathrm{x}-, \mathrm{y}$ -, and $\mathrm{z}$ - direction are equal each other:

$$
\left\langle\mathrm{x}^{2}\right\rangle=\left\langle\mathrm{y}^{2}\right\rangle=\left\langle\mathrm{z}^{2}\right\rangle=\left\langle\mathrm{r}^{2}\right\rangle
$$

As a result, the expectation value of $\mathrm{r}^{2}=\mathrm{x}^{2}+\mathrm{y}^{2}+\mathrm{z}^{2}$ is

$$
\left\langle\mathrm{r}^{2}\right\rangle=\left\langle\mathrm{y}^{2}+\mathrm{z}^{2}+\mathrm{x}^{2}\right\rangle=3\left\langle\mathrm{z}^{2}\right\rangle
$$

in the spherical case. Here $\left\langle\mathrm{r}^{2}\right\rangle$ is the mean square radius of the orbit.

The electric quadrupole moment $\mathrm{eQ}$, which measures the lowest order departure from a spherical charge distribution in a nucleus, is defined in terms of the difference between $3 z^{2}$ and $\mathrm{r}^{2}$ :

$$
e Q=e\left(3 z^{2}-r^{2}\right)
$$

For a spherical nucleus we have $\langle\mathrm{Q}\rangle=0$. For a cigar shaped (prolate) nucleus, the charge is concentrated along $\mathrm{z}$, and $\mathrm{Q}$ is positive [3].

The quadrupole moment of a disk shaped (oblate) nucleus $\mathrm{Q}$ is negative. A positive quadrupole moment of $\mathrm{Q}=0.29 \mathrm{fm}^{2}$ according experiment indicates that the deuteron is slightly elongated the $\mathrm{z}$ - axiz, like an olive (prolate). Quantum mechanical definition of quadrupole moment for a single proton [17] is described by:

$$
\mathrm{eQ}=\mathrm{E} \int \Psi^{*}\left(3 \mathrm{z}^{2}-\mathrm{r}^{2}\right) \Psi \mathrm{dt}
$$

Thus, if the quadrupole moment is not equal to zero then the eigenfunction of the ground state of the deuteron assigns a probability of 0.04 to finding a ${ }^{3} \mathrm{D}_{1}$ state and a probability of a 0.96 to finding a ${ }^{3} \mathrm{~S}_{1}$ state [2]. The last one points to the tensor character of the nucleon - nucleon interaction (the more details see, e.g. [5]). Nuclear magnetic dipole and electric quadrupole have a similar importance in helping us to interpret the deuteron structure. We should repeat that each nuclear state is assigned a unique "spin" quantum number I, representing the total angular momentum (orbital + intrinsic) of all the nucleons in the nucleus. Taking into account the algebra rules, vector $\overrightarrow{\mathrm{I}}$ is equal (see, e.g. [2]): 


$$
\overrightarrow{\mathrm{I}}=\sum_{\mathrm{i}=1}^{\mathrm{A}}\left(\overrightarrow{l_{\mathrm{i}}}+\overrightarrow{\mathrm{s}_{\mathrm{i}}}\right)=\overrightarrow{\mathrm{L}}+\overrightarrow{\mathrm{S}}
$$

here $\overrightarrow{l_{i}}$ and $\overrightarrow{s_{i}}$ orbital and spin, respectively of the $\mathrm{i}-\mathrm{nu}-$ cleon. Analogous picture we have for the total (orbital $\overrightarrow{l_{\mathrm{i}}}(\mathrm{e})$ and spin $\overrightarrow{\mathrm{s}_{\mathrm{i}}}(\mathrm{e})$ ) electronic angular momentum:

$$
\overrightarrow{\mathrm{J}}=\sum_{\mathrm{i}=1}^{\mathrm{Z}}\left(\overrightarrow{l_{\mathrm{i}}}(\mathrm{e})+\overrightarrow{\mathrm{s}_{\mathrm{i}}}(\mathrm{e})\right)
$$

Sum of the $\overrightarrow{\mathrm{I}}$ and the $\overrightarrow{\mathrm{J}}$ vectors determines the vector $\overrightarrow{\mathrm{F}}$ :

$$
\overrightarrow{\mathrm{F}}=\overrightarrow{\mathrm{I}}+\overrightarrow{\mathrm{J}} \text {. }
$$

We should underlined that all indicated vectors $\overrightarrow{\mathrm{F}}, \overrightarrow{\mathrm{I}}, \overrightarrow{\mathrm{J}}$ obey the usual quantum mechanics rules for angular momentum (see, e.g. [17]).

As is well - known, nuclear magnetic moment is the sum orbital and intrinsic spin moments, e.g.

$$
\mu=\left(\mathrm{g}_{l} 1+\mathrm{g}_{\mathrm{s}} \mathrm{s}\right) \mu_{\mathrm{N}} / \hbar
$$

here the nuclear $\mathrm{g}$ - factor is dimensionless number $\mathrm{g}_{l}=$ $\gamma \hbar / \mu_{N}$ (where $\gamma$ is gyromagnetic ratio $[1,2]$ ). In equation (9) $g_{l}$ and $g_{s}$ are the orbital and intrinsic contributions to $\mu$. Their values are even to $\mathrm{g}_{1}=1$ and $\mathrm{g}_{\mathrm{s}}=5.5856912$ for protons and $\mathrm{g}_{1}=0$, and $\mathrm{g}_{\mathrm{s}}=-3.8260837$ for uncharged neutrons. Today we haven't a single theory, which allows to calculate $\mu[18,19]$. Now relativistic quark models without gluonic or pion cloud degrees of freedom generally predict about $60 \%$ of the proton's spin should be carried by the quarks, with remaining $40 \%$ in quark orbital and angular momentum [19]. Today data and theory point to a consistent picture where the proton spin puzzle is a valence quark effect (the details see $[18,19])$.

Originally the hyperfine structure was taken to include those atomic effects (much smaller than the fine structure) that arise from the coupling between the electronic and nuclear angular moments. It is thus an internal effect in atoms, and we cannot switch it off or modify it except by changing the nuclear or electronic structure (going to excited states, for instance). The motion of the electrons produces a magnetic field $\overrightarrow{\mathrm{B}_{\mathrm{e}}}$ at the nucleus, which interacts with the nuclear magnetic moment $\mu_{\mathrm{N}}$ (see, e.g. [20]):

$$
\mathrm{E}=-\vec{\mu}_{N} \cdot \overrightarrow{\mathrm{B}}_{\mathrm{e}}
$$

Typical energy differences of hyperfine multiplets are only about $10^{-7} \mathrm{eV}$ (in case of the deuteron it is $3.16 \cdot 10^{-7} \mathrm{eV}$ ). This value is by more than seven order less than we observe in experiments: the isotopic shift of the $n=1$ s excitons is equal to $0.103 \mathrm{eV}$. The longitudinal character of electric charges of the $u$ and d quarks are the only mechanism of the renormalization of the energy elementary excitations (excitons, phonons) in studied crystals by the strong interaction but not very convincing one. The difference mass of the neutron and proton if determined mainly exactly by the difference of mass of $u$ and d quarks [21, 22]. Thus observed effects in experiments don't find consistent explanation at the change strong interaction leaving it to be another mystery of Standard Model (see, also [4, 21]). We should remind that intrinsic contradiction of Standard Model is already well known. Really, the Lagrangian of quantum chromodynamics (theory of the strong interaction) has the next form (see, e.g. [23]):

$$
\mathrm{L}=\mathrm{i} \sum_{\mathrm{q}} \bar{\Psi}_{\mathrm{q}}^{\mathrm{a}}\left(\nabla_{\mu} \gamma_{\mu}+\mathrm{im}_{\mathrm{q}}\right) \Psi_{\mathrm{q}}^{\mathrm{a}}-\frac{1}{4} \mathrm{G}_{\mu \nu}^{\mathrm{n}} \mathrm{G}_{\mu \nu}^{\mathrm{n}},
$$

where

$$
\begin{gathered}
\nabla_{\mu}=\partial_{\mu}-\operatorname{ig} \frac{\lambda^{\mathrm{n}}}{2} \mathrm{~A}_{\mu}^{\mathrm{n}}, \\
\mathrm{G}_{\mu \nu}^{\mathrm{n}}=\partial_{\mu} \mathrm{A}_{\mu}^{\mathrm{n}}-\partial_{\mathrm{n}} \mathrm{A}_{\mu}^{\mathrm{n}}+\mathrm{gf}^{\mathrm{nml}} \mathrm{A}_{\mu}^{\mathrm{n}} \mathrm{A}_{\nu}^{l} .
\end{gathered}
$$

$\Psi_{\mathrm{q}}^{\mathrm{a}}$ and $\mathrm{A}_{\mu}^{\mathrm{n}}$ are quark and gluon fields, $\mathrm{a}=1,2,3, \ldots 8$ are color indices , $\lambda^{\mathrm{n}}$ and $\mathrm{f}^{\mathrm{nml}}$ are Gell - Mann matrices and $\mathrm{f}$ symbols, $\mathrm{m}_{\mathrm{q}}$ - are bare (current) masses, $\mathrm{q}=\mathrm{u}, \mathrm{d}, \mathrm{s}, \mathrm{c}, \ldots$ different quarks. It is common place [4, 24] that the Lagrangian (11) contains the members which describe both free motion and interaction between quarks and gluons, which is defined by the strength couple g. Spacing of which it is necessary to remark that although the Lagrangian (8) possesses rather attractive peculiarities (see, also $[4,20]$ ), its eigenstates are the quarks and the gluons which are not observed in free states [24]. The observed hadrons in the experiment don't eigenstates in quantum chromodynamics. It is obvious to expect that the modern theory of quantum chromodynamics should finally overcome these difficulties [23-24]. We should add that the current theoretical and experimental evidence for the existence of electronic objects with a fractional of electron charge $(\mathrm{e} / 2, \mathrm{e} / 3$, etc) is reviewed in paper [25].

\section{Conclusion}

The experimental evidence of the macroscopic manifestation of strong (nuclear) interaction in optical spectra of solids which are differ by term of one neutron from each other has been presented for the first time. This evidence is based on two independent experimental results, which is directly seen from luminescence and scattering spectra. As far as the gravitation, electromagnetic and weak interactions are the same in both of kind crystals, it only changes the strong interaction. Therefore a logical conclusion is made that the renormalization of the energy of electromagnetic excitations (excitons, phonons) is carried out by the strong (nuclear) interaction. There is underlined the necessity consideration the strong (nuclear) interaction in quantum electrodynamics.

\section{REFERENCES}

[1] Ju. M. Shirokov, N.P. Judin, Nuclear Physics (Science, Moscow, 1980) (in Russian).

[2] K.S. Krane, Introductory Nuclear Physics (John Wiley \& Sons, New York, Chichester, 1988). 
[3] V.G. Plekhanov, Isotopes in Condensed Matter (Springer, Heidelberg - Berlin, 2013).

[4] F.I. Yndurain, The Theory of Quark and Gluon Interactions (Springer, Berlin, 1999).

[5] E. Segre, Nuclei and Particles (W.A. Benjamin, Inc. , Reading, MA, 1977).

[6] V.G. Plekhanov and V.I. Altukhov, Light scatteting in LiH crystals with LO phonon emission, J. Raman Spectr. 16 (1985) 358.

[7] V.G. Plekhanov Experimental manifestation of the effect of disorder on exciton binding energy in mixed crystals, Phys. Rev. B53 (1996) 9558.

[8] V.G. Plekhanov, Fundamentals and applications of isotope effect in solids, Prog. Mat. Sci. 51 (2006) 287.

[9] O.I. Tuytuynnik, V.I. Tuytuynnik, F.F. Gavrilov and G.I. Pilipenko, Lithium hydride single crystal growth by Bridgmen - Stockbarger method using ultrasound, J. Crystal Growth 68 (1984) 741 .

[10] V.G. Plekhanov, Isotopic and disorder effects in large radius exciton spectroscopy, Phys. - Uspekhi 40 (1997) 553.

[11] R.S. Knox, Theory of Excitons (Academic Press, New York London, 1963).

[12] S. Baroni, G. Pastori Parravicini, G. Pezzics, Quasiparticle band structure of lithium hydride, Phys. Rev. B32 (1985) 4077

[13] J.L. Verble, J.L. Warren and J.L. Yarnell, Latice dynamics of lithium hydride, ibid, 168 (1968) 980 .

[14] V.G. Plekhanov, Fundamentals and applications of isotope effect in modern technology, J. Nucl. Sci. \& Technol. (Japan) 43 (2006) 375.
[15] V.G. Plekhanov, Isotope effects in lattice dynamics, Phys. Uspekhi 46 (2003) 689.

[16] J Carlson R. Schivalia, Structure and dynamics of few - nucleon systems, Rev. Mod. Phys. 70 (1998) 743.

[17] R.N. Zare, Angular Momentum (John Wiley \& Sons, New York - Chichester, 1988).

[18] S.D. Bass, The spin structure of the proton, Rev. Mod. Phys. 77, (2005) 1257 - 1289

[19] C.A. Aidala, S.D. Bass, D. Hasey, The spin structure of the nucleon, ArXiv: hep - ph/1209/2803 (2013), Rev. Mod. Phys. 85 (2013) 655 - 691

[20] H. Haken, H. Ch. Wolf, The Physics of Atoms and Quanta (Springer, Berlin, 2005).

[21] G.A. Miller, Charge densities of the neutron and proton, Phys. Rev. Lett. 99 (2007)112001 - 4.

[22] V.G. Plekhanov, The enigma of the mass, ArXiv: phys/0906.4408 (2009).

[23] B.L. Ioffe, QCD at low energy, Prog. Part. Nucl. Phys. 56 (2006) 232 - 257

[24] I.M. Dremin, A.B. Kaidalov, Quantum chromodynamics and the phenomenology of strong interaction, UFN (Moscow) 176 (2006) 275 (in Russian), Phys. - Uspekhi 49 (2006) 263 - 273

[25] V.P. Bykov, Fractional charge: a new trend in electronics, UFN (Moscow) 176 (2006) 1007 (in Russian), Phys. Uspekhi 49 (2006) 979 - 985. 